\title{
Laminoplasty versus laminectomy and fusion for multilevel cervical myelopathy: a meta-analysis of clinical and radiological outcomes
}

\author{
Chang-Hyun Lee, MD, ${ }^{1,2}$ Jaebong Lee, MSc, ${ }^{3}$ James D. Kang, MD, ${ }^{4}$ Seung-Jae Hyun, MD, ${ }^{1}$ \\ Ki-Jeong Kim, MD, ${ }^{1}$ Tae-Ahn Jahng, MD, ${ }^{1}$ and Hyun-Jib Kim, MD ${ }^{1}$ \\ 'Department of Neurosurgery, Spine Center, Seoul National University Bundang Hospital, Seoul National University College \\ of Medicine, Seongnam; '2Department of Neurosurgery, Konyang University Hospital, Konyang University College of Medicine, \\ Daejeon; ${ }^{3}$ Medical Research Collaborating Center, Seoul National University Bundang Hospital, Seongnam, Republic of Korea; \\ and ${ }^{4}$ Department of Orthopaedic Surgery, University of Pittsburgh School of Medicine, Pittsburgh, Pennsylvania
}

OBJECT Posterior cervical surgery, expansive laminoplasty (EL) or laminectomy followed by fusion (LF), is usually performed in patients with multilevel ( $\geq 3$ ) cervical spondylotic myelopathy (CSM). However, the superiority of either of these techniques is still open to debate. The aim of this study was to compare clinical outcomes and postoperative kyphosis in patients undergoing EL versus LF by performing a meta-analysis.

METHODS Included in the meta-analysis were all studies of EL versus LF in adults with multilevel CSM in MEDLINE (PubMed), EMBASE, and the Cochrane library. A random-effects model was applied to pool data using the mean difference (MD) for continuous outcomes, such as the Japanese Orthopaedic Association (JOA) grade, the cervical curvature index (CCl), and the visual analog scale (VAS) score for neck pain.

RESULTS Seven studies comprising 302 and 290 patients treated with EL and LF, respectively, were included in the final analyses. Both treatment groups showed slight cervical lordosis and moderate neck pain in the baseline state. Both groups were similarly improved in JOA grade (MD 0.09, $95 \% \mathrm{Cl}-0.37$ to $0.54, p=0.07$ ) and neck pain VAS score (MD $-0.33,95 \% \mathrm{Cl}-1.50$ to $0.84, p=0.58$ ). Both groups evenly lost cervical lordosis. In the LF group lordosis seemed to be preserved in long-term follow-up studies, although the difference between the 2 treatment groups was not statistically significant.

CONCLUSIONS Both EL and LF lead to clinical improvement and loss of lordosis evenly. There is no evidence to support EL over LF in the treatment of multilevel CSM. Any superiority between EL and LF remains in question, although the LF group shows favorable long-term results.

http://thejns.org/doi/abs/10.3171/2014.10.SPINE1498

KEY WORDS myelopathy; laminectomy; laminoplasty; fusion; meta-analysis; comparison; cervical

$\mathrm{C}$ ERVICAL spondylotic myelopathy (CSM) is the leading cause of spinal cord dysfunction. ${ }^{17}$ The spinal cord in CSM is compressed because of the degeneration of intervertebral discs and the secondary degeneration of stable structures, such as the uncovertebral joint, facet joint, posterior longitudinal ligament, and ligamentum flavum. ${ }^{4}$ Because compressive lesions leading to cervical myelopathy usually arise anteriorly, proponents of anterior surgery cite as advantages the ability to directly remove the majority of compressive lesions in the cervical spine, a muscle-sparing dissection, which results in minimal postoperative pain, and the ability to correct and decompress the cord over kyphotic lesions. ${ }^{13}$ In CSM patients with more than 3 affected spinal cord segments, the posterior approach looks attractive given the accelerated complication rates associated with anterior surgery and especially fusion-related problems with long strut grafts to reconstruct multilevel corpectomies. ${ }^{13}$

ABBREVIATIONS CCI = cervical curvature index; CSM = cervical spondylotic myelopathy; EL = expansive laminoplasty; JOA = Japanese Orthopaedic Association; LF = laminectomy followed by fusion; MD = mean difference; VAS = visual analog scale.

SUBMITTED January 29, 2014. ACCEPTED October 21, 2014.

INCLUDE WHEN CITING Published online March 27, 2015; DOI: 10.3171/2014.10.SPINE1498.

DISCLOSURE The authors report no conflict of interest concerning the materials or methods used in this study or the findings specified in this paper. Dr. Lee received funding for this study from AOSpine Korea (AOSKR[R] 2014-05), a nonprofit foundation for spine education and research. Dr. Kang has received support from Synthes for non-study-related clinical or research effort. 
The posterior surgery techniques for cervical myelopathy have considerably evolved over the past 40 years. In the past, decompressive laminectomy alone was regarded as the gold-standard treatment for multilevel cervical myelopathy. However, postlaminectomy complications, such as kyphosis and segmental instability, have required additional fixation using lateral mass screws. ${ }^{9,17}$ Currently, laminectomy followed by lateral mass screw placement and fusion (LF) or expansive laminoplasty (EL) is usually performed as a posterior cervical surgery for CSM. Laminectomy followed by fusion is associated with low rates of kyphosis and segmental instability as compared with those for EL. However, LF alters normal cervical biomechanics, as axial and rotational forces are no longer physiologically distributed to subjacent spinal structures.$^{17}$ Expansive laminoplasty preserves motion with less substantial alterations to the natural biomechanics of the cervical spine. In turn, EL creates less extensive cord decompression as compared with laminectomy and reduces the range of motion to approximately $40 \% .{ }^{8}$

Previous studies have demonstrated favorable clinical outcomes after LF, but not after $\mathrm{EL}^{7,17}$ or vice versa. ${ }^{5,11}$ Other studies addressing the superiority of one technique over the other have shown controversial results.,12,18,19 The aim of the present study was to compare improvements in myelopathy, the development of postoperative kyphosis, and the prevalence of axial pain in patients undergoing EL or LF by using a meta-analysis.

\section{Methods}

\section{Information Sources}

Eligible studies had to include adults with CSM who had undergone EL or LF. Our outcomes of interest were as follows: neck pain, functionality, kyphosis. We performed a computerized search of the MEDLINE (PubMed; 1950 to July 2013) and EMBASE (1980 to July 2013) databases and the Cochrane Central Register of Controlled Trials (Issue 7 of 12 July 2013). Eligible studies had to include the words "cervical," "laminectomy," and "laminoplasty." We restricted the search to adult humans but not to any specific language(s). We removed duplicate articles and reviewed titles and abstracts. Studies meeting the following criteria were included in this analysis: 1) those comparing EL with LF; 2) those including patients with CSM caused by multisegmental spinal stenosis ( $\geq 3$ segments); and 3) those comprising patients who underwent EL or LF. We also searched the bibliographies of relevant articles to identify additional studies. A paper "in press" was included..$^{10}$

Two reviewers independently assessed the eligibility of all studies retrieved from the databases. Any disagreement between the reviewers was resolved through discussion. From the studies included in the final analysis, we extracted the following information: 1) study design; 2) country of origin; 3) patient demographics; 4) length of follow-up; 5) patient distribution for each group; 6) mean (and standard deviation) Japanese Orthopaedic Association (JOA) grade before and after posterior surgeries; 7) mean (and standard deviation) visual analog scale (VAS) score before and after the surgeries; and 8) mean (and standard deviation) cervical curvature index (CCI), also referred to as the Ishihara index, before and after the surgeries. When necessary, we contacted corresponding authors of the eligible studies to obtain additional data or clarification..$^{10,17} \mathrm{We}$ assessed the bias risk using the Cochrane Collaboration's "risk of bias" tool in a randomized controlled study and a modified form of the Newcastle-Ottawa Scale for nonran-

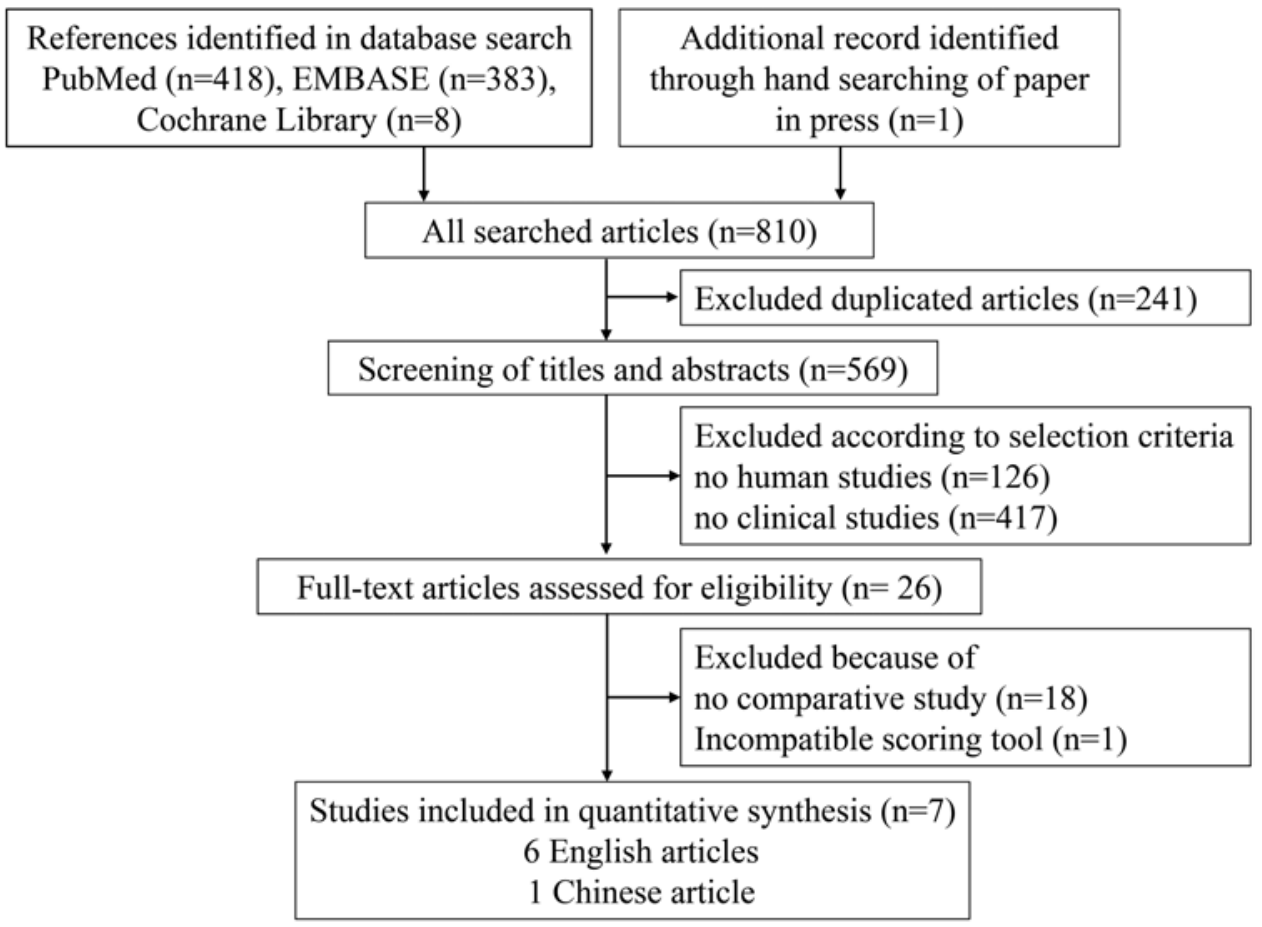

FIG. 1. Flowchart for the identification of relevant studies. 
domized studies. ${ }^{6,16}$ At least 2 researchers independently assessed the risk of bias, with disagreements resolved through discussion.

\section{Statistical Analyses}

To analyze surgical effectiveness via continuously distributed outcomes (JOA grade, VAS score, and CCI), we calculated the mean differences (MDs) between scores obtained preoperatively and those obtained postoperatively in both the EL and the LF groups. We estimated the MD with a 95\% confidence interval (CI) based on both fixed and random-effects models. Because we observed high heterogeneity in most analyses, we reported the MD with the 95\% CI based on random-effects models. A funnel plot was constructed and the Egger test was performed to assess for the presence of publication bias. All statistical tests were performed using R software (version 3.0.1, The $\mathrm{R}$ Foundation for Statistical Computing). A p value $<0.05$ was considered to indicate statistical significance.

\section{Results}

\section{Search Results for Relevant Studies}

An initial search using the subject headings identified 418 studies in MEDLINE, 383 studies in EMBASE, and 8 studies in the Cochrane Central Register of Controlled Trials. One paper was identified through hand searching. ${ }^{10}$ Among these 810 studies, 241 duplicated articles were excluded. Among the 569 remaining studies, 126 did not deal with human subjects and 417 papers were case reports, technical notes, or review articles; therefore, these 543 papers were excluded after reviewing article titles and abstracts. The remaining 26 studies were subjected to a full-text review. An additional 19 studies were subsequently excluded as these analyses were not comparative studies (18 papers) or they used an incompatible scoring tool (1 paper). Finally, a total of 7 studies were included in the meta-analysis (6 English papers and 1 Chinese paper)..$^{2,7,10,11,17-19}$ The detailed selection process is shown in Fig. 1.

The 7 studies comprised a total of 592 patients (302 patients in the EL group, 290 patients in the LF group). The mean ages were 56.2 and 58.9 years in the EL and LF groups, respectively. Approximately $68 \%$ and $64.4 \%$ of the patients were male in the EL and LF groups, respectively. Table 1 shows general information about the 7 studies included in this analysis. The countries in which the studies were conducted included the United States $(n=3)$, China $(n=3)$, and the Republic of Korea $(n=1)$. Because the EL and the LF groups in comparative studies matched in terms of cervical lordosis and neck disability, the 2 groups did not show significant differences in JOA grade, VAS score, and CCI at the baseline state (Table 2). The average JOA score at baseline was 9.35 in the EL group and 9.31 in the LF group, while the preoperative VAS score was 3.83 and 3.67 , respectively. The average CCI at baseline was 5.29 in the EL group and 3.45 in the LF group. The minimum follow-up period ranged from 6 months to 4 years.

\section{Comparison of 2 Posterior Surgeries}

Both the EL and LF groups showed improved JOA

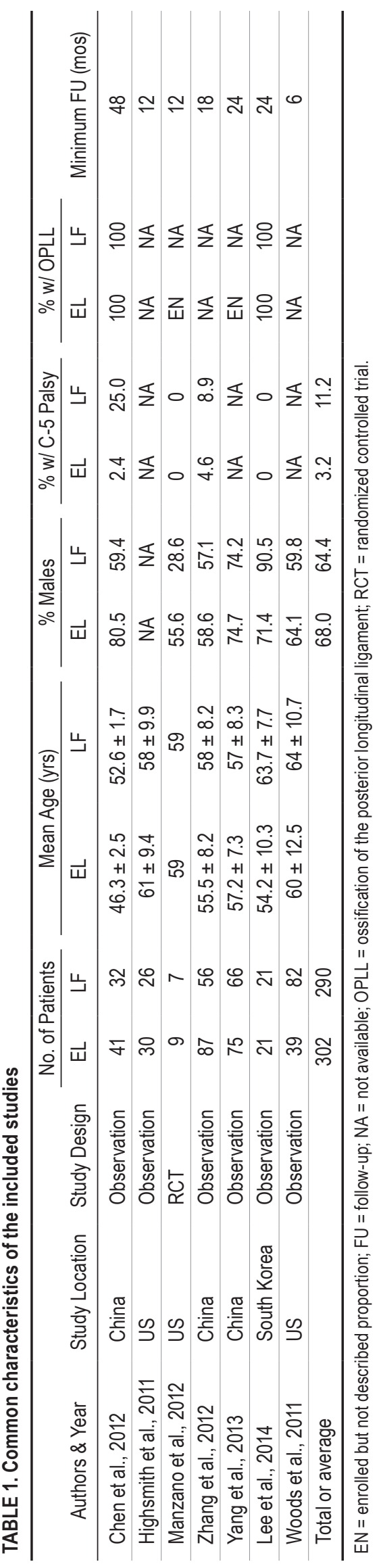

J Neurosurg Spine Volume 22 • June 2015 
C. H. Lee et al.

TABLE 2. End points of the studies included in the meta-analysis

\begin{tabular}{|c|c|c|c|c|c|c|c|}
\hline \multirow[b]{2}{*}{ Authors \& Year } & \multirow[b]{2}{*}{ State } & \multicolumn{2}{|c|}{$\mathrm{JOA}$} & \multicolumn{2}{|c|}{ VAS } & \multicolumn{2}{|c|}{$\mathrm{CCl}$} \\
\hline & & $E L$ & LF & EL & LF & $\mathrm{EL}$ & LF \\
\hline \multirow[t]{2}{*}{ Chen et al., 2012} & Baseline & $10.2 \pm 0.3$ & $9.1 \pm 0.4$ & NA & NA & NA & NA \\
\hline & Final & $14.6 \pm 0.2$ & $13 \pm 0.2$ & NA & NA & NA & NA \\
\hline \multirow[t]{2}{*}{ Highsmith et al., 2011} & Baseline & $11.6 \pm 2.6$ & $12.4 \pm 2.7$ & $3.2 \pm 2.8$ & $5.8 \pm 3.2$ & NA & NA \\
\hline & Final & $14.4 \pm 2.3$ & $15.2 \pm 1.9$ & $3.4 \pm 2.6$ & $3.0 \pm 2.3$ & NA & NA \\
\hline \multirow[t]{2}{*}{ Manzano et al., 2012} & Baseline & $12.4 \pm 1.2$ & $12.6 \pm 1.1$ & $1.8 \pm 0.2$ & $2.7 \pm 0.6$ & -0.2 & -1.9 \\
\hline & Final & $14.3 \pm 1.0$ & $13.6 \pm 1.0$ & $0.7 \pm 0.4$ & $2.2 \pm 0.8$ & $-4.6 \pm 4.8$ & $-6.3 \pm 4.7$ \\
\hline \multirow[t]{2}{*}{ Zhang et al., 2012} & Baseline & $7.1 \pm 0.9$ & $7.2 \pm 1.1$ & $5.2 \pm 2.8$ & $4.8 \pm 3.2$ & $11.3 \pm 1.8$ & $11 \pm 2.3$ \\
\hline & Final & $13.6 \pm 1.2$ & $13.8 \pm 1.0$ & $2.8 \pm 2.6$ & $2.0 \pm 2.3$ & $9.8 \pm 2.2$ & $10.6 \pm 1.8$ \\
\hline \multirow[t]{2}{*}{ Yang et al., 2013} & Baseline & $8.91 \pm 1.23$ & $8.65 \pm 1.05$ & $2.85 \pm 1.11$ & $2.59 \pm 1.25$ & $0.15 \pm 0.06$ & $0.15 \pm 0.05$ \\
\hline & Final & $13.55 \pm 1.34$ & $13.59 \pm 1.08$ & $1.11 \pm 1.18$ & $2.15 \pm 1.38$ & $0.14 \pm 0.06$ & $0.14 \pm 0.06$ \\
\hline \multirow[t]{2}{*}{ Lee et al., 2014} & Baseline & $14.0 \pm 2.8$ & $12.4 \pm 2.9$ & $3.4 \pm 3.5$ & $2.9 \pm 2.8$ & $10.9 \pm 6.5$ & $8.4 \pm 8.3$ \\
\hline & Final & $13.6 \pm 3.4$ & $13.1 \pm 1.2$ & $2.9 \pm 2.8$ & $1.3 \pm 1.7$ & $5.7 \pm 6.4$ & $5.3 \pm 8.9$ \\
\hline \multirow[t]{2}{*}{ Woods et al., 2011} & Baseline & NA & NA & NA & NA & $0.17 \pm 0.03$ & $0.13 \pm 0.05$ \\
\hline & Final & NA & NA & NA & NA & $0.18 \pm 0.07$ & $0.10 \pm 0.02$ \\
\hline
\end{tabular}

JOA

Study

Chen, 2012

Highsmith, 2011

Manzano, 2012

Zhang, 2012

Yang, 2013

Lee, 2014

Random effects model 263

Heterogeneity: l-squared $=79.6 \%$, tau-squared $=0.1902, p=0.0002$

\section{Laminoplasty Laminectomy+Fusion Mean difference \\ Total Mean SD Total Mean SD}

$\begin{array}{rrrrrr}41 & 4.40 & 0.26 & 32 & 3.90 & 0.35 \\ 30 & 2.80 & 2.46 & 26 & 2.80 & 2.40 \\ 9 & 1.90 & 1.11 & 7 & 1.00 & 1.05 \\ 87 & 6.50 & 1.08 & 56 & 6.60 & 1.05 \\ 75 & 4.64 & 1.29 & 66 & 4.94 & 1.07 \\ 21 & 1.20 & 3.14 & 21 & 2.20 & 2.52\end{array}$

208

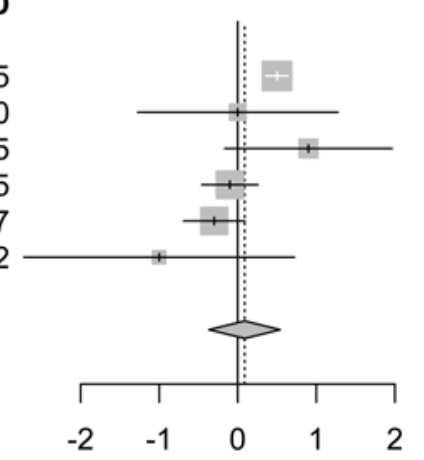

Laminectomy+Fusion Laminoplasty

$\begin{array}{rrr}\text { MD } & \mathbf{9 5 \% - C l ~ W ( r a n d o m ) ~} \\ & & \\ 0.50 & {[0.35 ; 0.65]} & 27.4 \% \\ 0.00 & {[-1.28 ; 1.28]} & 8.7 \% \\ 0.90 & {[-0.16 ; 1.96]} & 11.1 \% \\ -0.10 & {[-0.46 ; 0.26]} & 24.0 \% \\ -0.30 & {[-0.69 ; 0.09]} & 23.3 \% \\ -1.00 & {[-2.72 ; 0.72]} & 5.6 \% \\ & & \\ \mathbf{0 . 0 9} & {[-\mathbf{0 . 3 7} \mathbf{0 . 5 4}]} & \mathbf{1 0 0 \%}\end{array}$

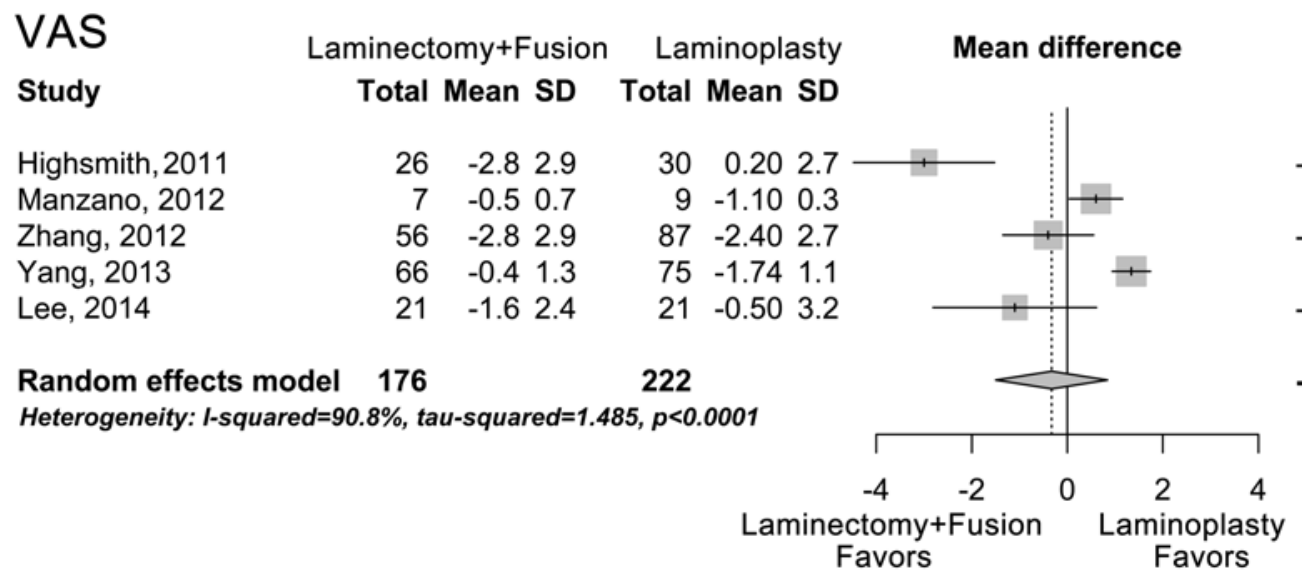

VAS

FIG. 2. Mean differences in changes in JOA (upper) and VAS (lower) scores after EL and LF in the random-effects meta-analysis. Both groups show a slight difference in JOA and VAS scores, although the difference was not statistically significant.

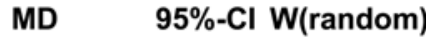

$-3.00 \quad[-4.48 ;-1.52] \quad 17.4 \%$

$0.60 \quad[0.05 ; 1.15] \quad 22.8 \%$

$-0.40 \quad[-1.35 ; 0.55] \quad 20.7 \%$

$1.34[0.94 ; 1.74] \quad 23.3 \%$

$-1.10[-2.81 ; 0.61] \quad 15.8 \%$

$-0.33[-1.50 ; 0.84] \quad 100 \%$ 
scores from 9.35 to 13.86 and from 9.31 to 13.71 , respectively. In the random-effects meta-analysis of 6 studies, no significant difference in the JOA score was observed between the 2 surgical methods, as shown in Fig. 2 (MD $0.09,95 \%$ CI -0.37 to $0.54, \mathrm{p}=0.07, \mathrm{I}^{2}=79.6 \%$ ). Axial neck pain VAS in the EL and LF groups improved similarly from 3.83 to 2.23 and from 3.67 to 2.13 , respectively. The meta-analysis revealed that the VAS score for the EL group was more improved than that for the LF group, as shown in Fig. 2 (MD -0.33, 95\% CI -1.50 to 0.84, p = 0.58) with high heterogeneity $\left(\mathrm{I}^{2}=90.8 \%\right)$. Because of publication bias, analysis of the VAS score may diminish the reliability of the results ( $p=0.02$, Egger test).

Two analyses of CCI were performed: one for all studies with available data (5 studies) 8 and another including only the studies with a reported follow-up of at least 18 months (3 studies). The CCI of the EL and LF groups changed from 5.29 to 4.08 and from 3.45 to 2.92 , respectively (Table 2 ). In the studies with follow-ups $\geq 18$ months, the CCI decreased -2.04 in the EL group and -1.05 in the LF group. There was no statistically significant difference in lordotic alignment preservation between the EL and LF groups, as shown in Fig. 3 (MD $-0.01,95 \%$ CI -0.09 to $0.07, \mathrm{p}=0.85$ ). Because of high heterogeneity, we applied the random-effects model $\left(I^{2}=88.2 \%\right)$. As postoperative cervical kyphosis is a long-term complication, studies with follow-ups $<18$ months were excluded. Subgroup analysis showed that the CCI for the LF group was reduced less than that for the EL group (MD -0.98, 95\% CI -2.20 to $0.23, \mathrm{p}=0.112)$ with high heterogeneity $\left(\mathrm{I}^{2}=92.2 \%\right)$. Although the difference in the decrease in CCI between the 2 groups increased in the long term, that difference was not statistically significant.

\section{Publication Bias}

Funnel plots were constructed for changes in JOA, VAS, and CCI in Fig. 4. The Egger test was calculated as $-1.64(\mathrm{p}=0.27),-5.70(\mathrm{p}=0.02)$, and $-2.37(\mathrm{p}=0.19)$ for JOA, VAS, and CCI, respectively. The funnel plots demonstrated a slight asymmetry in JOA and CCI, which indicated a reliable analysis. However, the funnel plot for VAS demonstrated substantial asymmetry, and the Egger test revealed substantial bias $(\mathrm{p}=0.02)$, which may indicate an underpowered analysis.

\section{Discussion}

Although both EL and LF are known to be safe and effective treatments for CSM in the neutral cervical spine, we found that LF is usually performed in North America. ${ }^{15,17}$ In contrast, Asian spine surgeons developed EL and usually prefer it. Strict indications for LF and EL differ according to cervical lordosis and instability. ${ }^{13}$ However, a fair number of patients showed a neutral cervical spine,

\section{Overall}

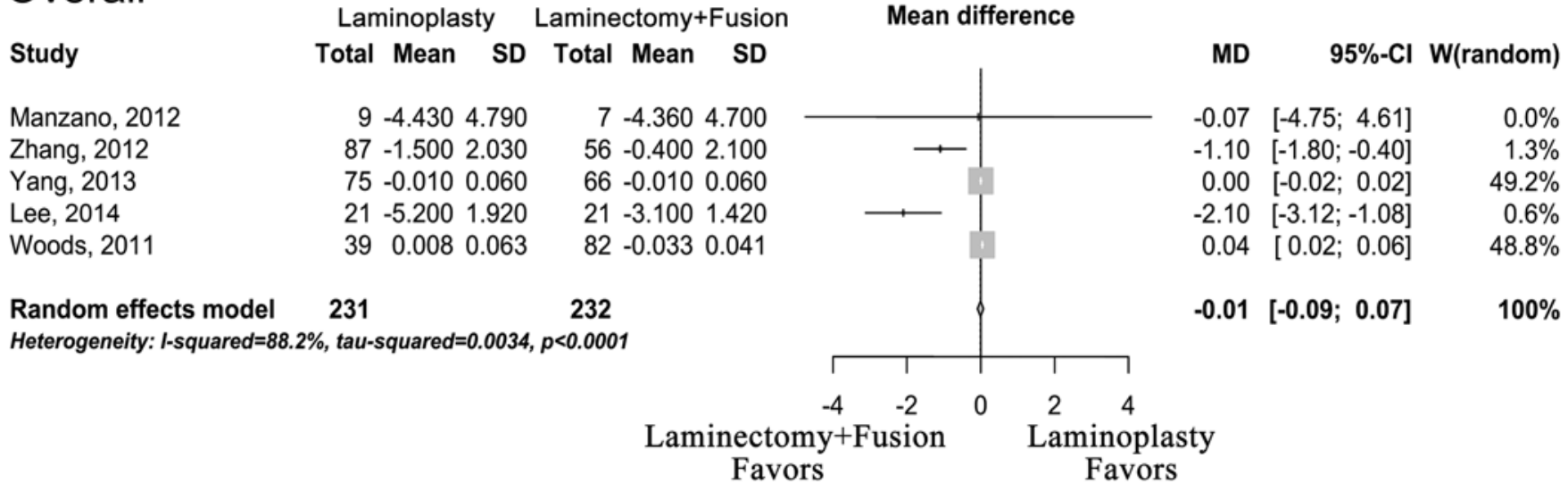

\section{8-month minimum follow-up}

Laminoplasty Laminectomy+Fusion

Study

Zhang, 2012

Yang, 2013

Lee, 2014

Random effects model 183

Total Mean SD

Laminectomy+Fusion
Total Mean SD

$\begin{array}{lll}87 & -1.50 & 2.03 \\ 75 & -0.01 & 0.06 \\ 21 & -5.20 & 1.92\end{array}$

Heterogeneity: $I$-squared $=92.2 \%$, tau-squared $=1.026, p<0.0001$
$\begin{array}{lll}56 & -0.402 .10\end{array}$
$\begin{array}{lll}66 & -0.01 & 0.06\end{array}$
$21-3.101 .42$

143

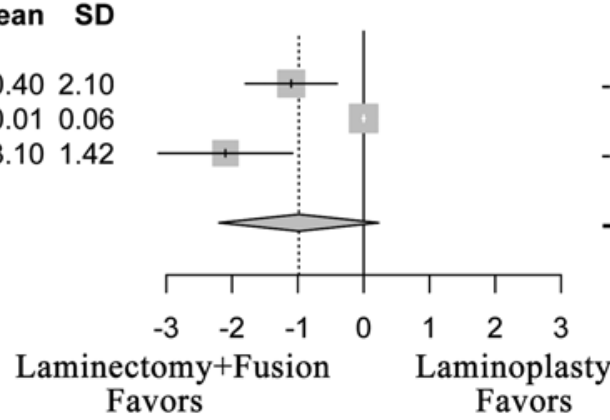

Laminectomy+Fusion Laminoplasty
Mean difference
MD

$95 \%-\mathrm{Cl}$ W(random)

$-1.10[-1.80 ;-0.40] \quad 33.2 \%$

$0.00[-0.02 ; 0.02] \quad 37.3 \%$

$-2.10[-3.12 ;-1.08] \quad 29.5 \%$

$-0.98[-2.20 ; 0.23] \quad 100 \%$

FIG. 3. Mean differences in changes in CCI between the EL and LF groups. All enrolled studies (upper) showed a similar loss of cervical lordosis. The subgroup analysis of studies with follow-up periods $\geq 18$ months (lower) showed increased rates of loss of lordosis with EL. SD = standard deviation; $\mathrm{W}=$ weight. 

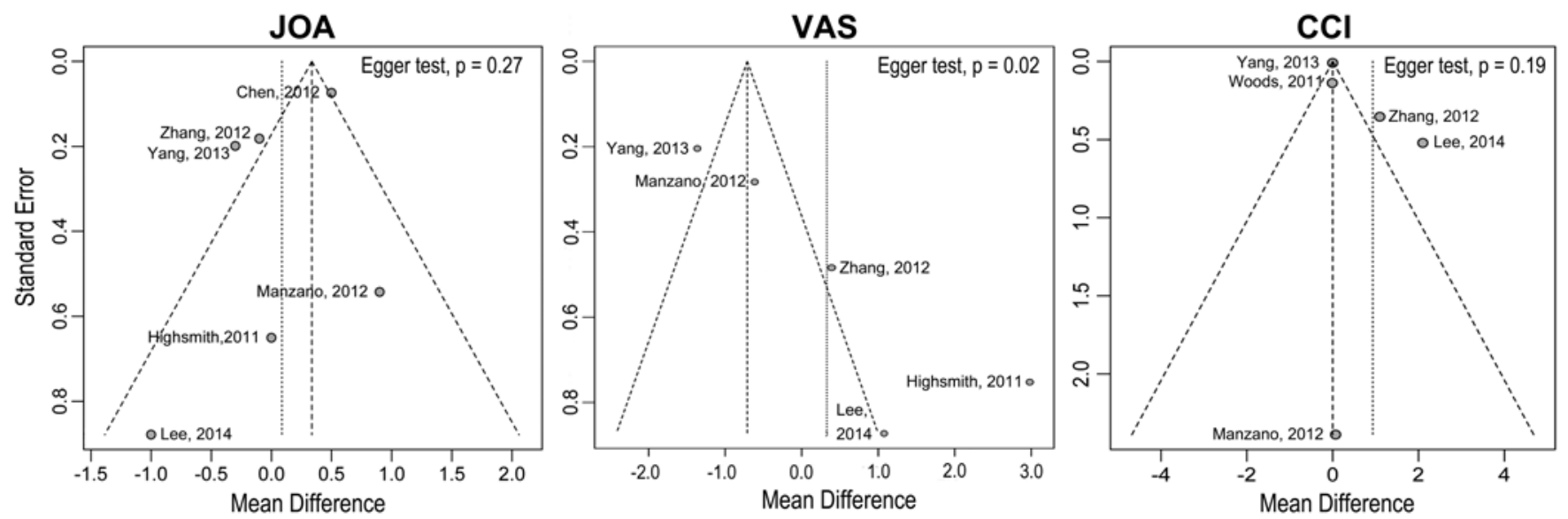

FIG. 4. A funnel plot and the Egger test demonstrated no substantial publication bias as regards the JOA grade and the $\mathrm{CCl}$ (JOA: $p=0.27 ; \mathrm{CCl}: p=0.19)$. However, the funnel plot and Egger test of the VAS score demonstrated substantial asymmetry $(p=0.02)$, which may indicate an underpowered analysis.

and there was no priority between EL and LF. Some investigators have demonstrated that laminoplasty can be performed with acceptable neurological recovery when local kyphosis measures $\leq 13^{\circ}$ preoperatively, ${ }^{14}$ whereas others have shown that laminoplasty is not routinely recommended for patients who do not have at least neutral to slightly lordotic alignment preoperatively. ${ }^{13}$

In this meta-analysis, all enrolled studies matched patients and showed similar scores on the JOA scale, VAS, and $\mathrm{CCI}$ in the 2 treatment groups at baseline. A majority of the patients revealed neutral to slightly lordotic cervical angles, mild to moderate myelopathy, and moderate neck pain. Both groups showed similar improvements in myelopathy, according to the JOA score, and in neck pain. This study showed no clear superiority between the EL and LF groups after surgery according to the JOA and neck pain VAS scores.

However, some studies reported opposite results in both the JOA and the VAS analyses; some studies favored EL, whereas others favored LF. There are a few potential explanations for the contrasting results, including the different effects of surgery in different patient populations, small or poor-quality studies, or random variation around a small true effect, among others. However, one might expect that, with an appropriate diagnosis and adequate decompression, similar average improvements on the JOA scale would be observed. On the other hand, the VAS pain scores are likely to be more sensitive to differences in patient populations.

The overall sagittal alignment changed to kyphosis, regardless of the surgical method. There was no substantial difference between the EL and LF groups. In the subgroup analysis of studies with follow-up periods of 18 months or more, the LF group may have had results superior to those in the EL group in terms of preserving lordosis in the long term. This different result in the subgroup analysis was caused by the relative weighting of the studies in the overall versus the long-term follow-up analysis. In the overall analysis, the studies of Zhang et al. ${ }^{19}$ and Lee et al. ${ }^{10}$ had very low weights (less than $2 \%$ for each), whereas in the long-term follow-up analysis they were weighted almost equally with the study of Yang et al. ${ }^{18}$ (approximately $30 \%$ each). The reason for this difference was that the study by Woods et al., ${ }^{17}$ which had a very narrow standard deviation, was excluded in the long-term follow-up analysis. In the literature review, LF was also generally recommended over EL for patients who did not have at least neutral to slightly lordotic alignment preoperatively.,3,17

\section{Study Limitations}

Our study has some important limitations that must be considered when interpreting the results. Preoperative lordotic alignment was worse in the LF group than in the EL group. Although a significant difference in preoperative CCI was not observed, the surgical indication was slightly different. However, each comparative study already considered this point and included a matched patient group. As an additional limitation, EL has different techniques, such as open door and French door; however, these differences were not considered.

\section{Conclusions}

There is no strong evidence to support EL over LF in multilevel CSM patients with neutral lordotic alignment. Both EL and LF may result in clinical improvement and a similar loss of lordosis. The superiority of either EL or LF remains in question, although the LF group showed favorable results in preserving lordosis over the long term.

\section{References}

1. Bartels RH, van Tulder MW, Moojen WA, Arts MP, Peul WC: Laminoplasty and laminectomy for cervical sponydylotic myelopathy: a systematic review. Eur Spine J [epub ahead of print], 2013

2. Chen Y, Liu X, Chen D, Wang X, Yuan W: Surgical strategy for ossification of the posterior longitudinal ligament in the cervical spine. Orthopedics 35:e1231-e1237, 2012

3. Edwards CC II, Heller JG: Posterior approaches for the surgical treatment for multilevel cervical spondylotic myelopathy: laminoplasty versus laminectomy. Curr Opin Orthop 12:224-230, 2001 
4. Edwards CC II, Riew KD, Anderson PA, Hilibrand AS, Vaccaro AF: Cervical myelopathy. Current diagnostic and treatment strategies. Spine J 3:68-81, 2003

5. Hardman J, Graf O, Kouloumberis PE, Gao WH, Chan M, Roitberg BZ: Clinical and functional outcomes of laminoplasty and laminectomy. Neurol Res 32:416-420, 2010

6. Higgins JP, Altman DG, Gøtzsche PC, Jüni P, Moher D, Oxman AD, et al: The Cochrane Collaboration's tool for assessing risk of bias in randomised trials. BMJ 343:d5928, 2011

7. Highsmith JM, Dhall SS, Haid RW Jr, Rodts GE Jr, Mummaneni PV: Treatment of cervical stenotic myelopathy: a cost and outcome comparison of laminoplasty versus laminectomy and lateral mass fusion. J Neurosurg Spine 14:619-625, 2011

8. Hyun SJ, Riew KD, Rhim SC: Range of motion loss after cervical laminoplasty: a prospective study with minimum 5-year follow-up data. Spine J 13:384-390, 2013

9. Kumar VG, Rea GL, Mervis LJ, McGregor JM: Cervical spondylotic myelopathy: functional and radiographic longterm outcome after laminectomy and posterior fusion. Neurosurgery 44:771-778, 1999

10. Lee CH, Jahng TA, Hyun SJ, Kim KJ, Kim HJ: Expansive Laminoplasty vs. laminectomy alone vs. laminectomy and fusion for cervical ossification of the posterior longitudinal ligament: is there a difference in the clinical or radiologic outcome? J Spinal Disord Tech [epub ahead of print], 2014

11. Manzano GR, Casella G, Wang MY, Vanni S, Levi AD: A prospective, randomized trial comparing expansile cervical laminoplasty and cervical laminectomy and fusion for multilevel cervical myelopathy. Neurosurgery 70:264-277, 2012

12. Nurboja B, Kachramanoglou C, Choi D: Cervical laminectomy vs laminoplasty: is there a difference in outcome and postoperative pain? Neurosurgery 70:965-970, 2012

13. Rhee JM, Basra S: Posterior surgery for cervical myelopathy: laminectomy, laminectomy with fusion, and laminoplasty. Asian Spine J 2:114-126, 2008

14. Suda K, Abumi K, Ito M, Shono Y, Kaneda K, Fujiya M: Local kyphosis reduces surgical outcomes of expansive opendoor laminoplasty for cervical spondylotic myelopathy. Spine (Phila Pa 1976) 28:1258-1262, 2003
15. Wang SJ, Jiang SD, Jiang LS, Dai LY: Axial pain after posterior cervical spine surgery: a systematic review. Eur Spine J 20:185-194, 2011

16. Wells GA, Shea B, O'Connell D, Peterson J, Welch V, Losos M, et al: The Newcastle-Ottawa Scale (NOS) for assessing the quality of nonrandomised studies in meta-analyses. Ottawa Hospital Research Institute. (http://www.ohri.ca/programs/clinical_epidemiology/oxford.asp) [Accessed February 19, 2015]

17. Woods BI, Hohl J, Lee J, Donaldson W III, Kang J: Laminoplasty versus laminectomy and fusion for multilevel cervical spondylotic myelopathy. Clin Orthop Relat Res 469:688695, 2011

18. Yang L, Gu Y, Shi J, Gao R, Liu Y, Li J, et al: Modified plateonly open-door laminoplasty versus laminectomy and fusion for the treatment of cervical stenotic myelopathy. Orthopedics 36:e79-e87, 2013

19. Zhang H, Sun T, Lu S, Li Q, Yadav SK: [Comparison of effectiveness between laminoplasty and laminectomy decompression and fusion with internal fixation for cervical spondylotic myelopathy.] Zhongguo Xiu Fu Chong Jian Wai Ke Za Zhi 26:1191-1196, 2012 (Chinese)

\section{Author Contributions}

Conception and design: CH Lee, KJ Kim, Jahng. Acquisition of data: CH Lee, Hyun. Analysis and interpretation of data: $\mathrm{CH}$ Lee, Kang. Drafting the article: $\mathrm{CH}$ Lee. Critically revising the article: KJ Kim, Jahng, HJ Kim. Reviewed submitted version of manuscript: Jahng. Statistical analysis: CH Lee, J Lee. Administrative/ technical/material support: Kang, Hyun. Study supervision: $\mathrm{CH}$ Lee, Jahng, HJ Kim.

\section{Correspondence}

Chang-Hyun Lee, Department of Neurosurgery, Konyang University Hospital, Konyang University College of Medicine, Gwanjeodong-ro 158, Seo-gu, Daejeon, 302-718, Republic of Korea.email: imspinesurgeon@gmail.com. 